Journal of Sustainability Perspectives

journal homepage: https://ejournal2.undip.ac.id/index.php/isp/

\title{
The Impact of Mobility Policy and Modal Share to The University Community in Bologna
}

\author{
Roberto Battistini $i^{1,}$, Luciana Sacchetti ${ }^{2}$, Andrea Braschi ${ }^{3}$ \\ ${ }^{1,2,3}$ Alma Mater Studiorum - Università di Bologna Italy \\ *corresponding author: roberto.battistini2@unibo.it
}

Article Info

\author{
Received: \\ 15 March 2021 \\ Accepted: \\ 25 May 2021 \\ Published: \\ 1 August 2021 \\ DOI:
}

Presented in The $6^{\text {th }}$ International (Virtual) Workshop on UI GreenMetric World University Rankings (IWGM 2020)

\begin{abstract}
In the Twentieth century transportation has been one of the most impactful drivers for economic and social development, while nowadays, it appears as a major cause of environmental and social problems. Even worse, the more transportation has become a relevant part in daily life and business, the more it is blamed for problems such as traffic congestion, car accidents, social economic inequality, energy consumption and pollution. Transportation is in fact caught in a vicious circle. Urban planning is forced to reduce transition costs, transforming cities in a sort of social network, and in parallel to orient mobility towards sustainability, both capable to convey socioeconomic development. Within this context, the University of Bologna has chosen a sustainable approach for its Multi-campus territorial structure, applying several sustainable mobility policies regarding public transportation, cycling, electric/hybrid car fleet and walking accessibility. The paper purpose is to show significative links between sustainable policy application and modal share, using data from an online survey submitted to university staff and students. Even gender influence on modal share has been investigated.
\end{abstract}

\section{Keyword:}

Modal Share, transport integration, bicycle, Survey, Sustainability

\section{Introduction: the role of urban mobility in sustainability issues}

More than half of people in the world live in urban contexts. More than 5 billion is forecasted to live in cities in 2030 [1]. These are critical aspects for many reasons. First, human health issues in high density urban context bring dramatic consequences [2]. It has been recently demonstrated that i.e. subway, wastewater and residential garbage are positively connected with the covid-19 transmission [3]. Moreover, it is easy to deduce also other hyper-population negative impacts into urban spaces, as the need to have access to several transportation grows. Thus, inducing environmental pollution, traffic congestion, unequal access to information, privacy issues. All these variables directly act on the urgency to rethink the link between households-spaces-mobility. Before 2030, cities have to set a new vision of humanized urban space, able to overcome the modern paradox cited by Edward Geaser, in which the more the travel costs are reduced, the more the proximity 
costs are increased. In any case, the solution is the so-called transitions costs reduction. Transition costs are related to infrastructure and services. This implies the transformation of cities into social network that spread-out information on sustainable mobility [4]. What is needed, is a structural change of the territorial spaces-mobility system, in comparison to the recent adopted measures [5].

Furthermore, Climate Change represents another relevant issue that catalyzes strategic measures and carbon management practices in local government [6]. Transportation has been seen as one of the most impactful cause of climate change, responsible for $25 \%$ of $\mathrm{CO} 2$ emissions in urban area and 1/3 of the $\mathrm{CO} 2$ emissions in the Earth atmosphere [7], while noise and economy problems created by traffic are additional causes. In these terms, it is easy to recognize that urban sustainable mobility may play a fundamental role to counterbalance the climate impact.

Sustainable mobility is the application of the sustainable development concept to the movement of people and goods [8]. It requires that the next generations' ability to meet their needs will not be compromised by the present generation needs and by the natural resources' exploitation.

Nevertheless, transportation was not recognized as a distinct Sustainable Development Goal (SDG), perhaps due to the impossibility to present the sector as a single element, being divided in sub-systems that influence the global process [9]. Some elements of transportation are included in some SDGs, i.e. road safety, carbon emissions, accessibility and social equity, with special attention to the needs of those in vulnerable situations, women, children, people with disabilities and the elderly.

Some transportation topics are included in SDG 11 (Sustainable City and Community), SDG7 (Affordable and clean Energy) and SDG 9 (Building resilient infrastructures). A full application of the SDGs implies a structural change involving three processes: increasing, transition and transformation [10]. While the increasing process is characterized by few sequential changes without structural alterations, transformation is a shocking approach oriented to demolish the past. Only the transition process combines the concept of an evolutionary path that seek to improve the present conditions, being the Transition towards sustainability a long-term multi actors' approach [11].

To offer to a high-density urban context an environmentally friendly and energy efficient transportation system it deserves new indicators to capture economic, environmental and social aspects [12].

The modal share indicator analyzes the current mobility and foresees its evolution. It shows the share of people which use a specific transportation's modality (including cycling and walking) within the overall transport usage of an urban area. Modal share can be calculated for passenger and freight (logistics) transport based on different units, such as number of trips, volume, weight, passenger-km or tons-km. The modal share is typically displayed as a percentage value for each kind of transportation. Modal share can be measured for specific trip types (e.g. journeys to work) or for the total of all journeys taken in a city for a given period of time. A challenge that still needs to be addressed is accurately counting intermodal journeys [1].

Modal share is also a relevant driver for the setting of an urban community sustainable strategies and policies. It is an efficient indicator to monitor their efficiency and effectiveness in terms of sustainable goals reached. 


\section{Transportation and sustainability at University of Bologna}

The University of Bologna has a complex academic setting displayed through a multicampus architecture. This means that there are several campuses in different cities of the Emilia-Romagna region: Bologna, Imola, Forlì, Cesena, Ravenna and Rimini. Each campus has a different spatial distribution: from a campus with facilities and locations spread-out in the town historical center, to peripherical campuses placed in isolated surroundings like micro-citadel similar to the of Anglo-Saxons setting.

Unibo attracts more than 80,000 students and 6,000 staff including professors, researchers, PhD students and administrative personnel. This large community deserves a specific attention regarding its urban impact in terms of sustainability. Indeed, 80,000 students, most of them living outside city-campus, represent a city-user with an approach to life-style and housing similar to long-term tourists. Students aliment the local economy but also generate collateral effects such as garbage, energy consumption, traffic congestion and environmental pollution.

With the help of mobility management tools, through the lens of an urban impacting community, the University of Bologna Governance, since 2017 has considered how to contain these strong emissions and effects on its territory related to a high student density. And this without neglecting the student daily life limitations mentioned above.

The principal indicator used to foster political decisions has been the Modal share. The complex paradigm of sustainable mobility of the Unibo Multicampus demands and articulated offer of different mobility solutions. One of the most common measure applied in modern urbanized context is transportation integration; with the term "integration" we mean the temporal and spatial chance of using different transportation vehicles. In literature, transport integration has been defined as a "pull" measure, referring to the capacity to be attractive for mobility users. Integration brings the user perception of transport system as whole [13]. The University of Bologna has found interest in research intermodal measure that could be attractive for students.

Secondary, it has been focused on the intention to study the modal share by the intermodal perspective which means to exam the trip chain of transportation vehicle used which characterize any commuting journey.

Surveys are one of the most influential methods for data collection on individual choice, while, on the other hand, there are objections on the reliability of this statistical tool. From the perspective of the Social Science and the Cognitive Psychology, a survey represents a social microcosmos in which the interviewer elicits information from the respondent in a specific interaction's modality [14]. In this context, there are several ambiguities that effect response and consequent data uncertainty and reliability. Some of these cognitive distortions derive from the response alternatives, from the impact of intensifying words such as "very", "extremely" [15], [16], from the memory process and from influence of the hierarchical relationship between the interviewer and the respondent. Not to mention the errors linked to the consistency and structure of the sample and the default connected to the abandon rate.

A solid way to reduce survey limitations and statistical errors, is to integrate the survey results with observational evidences. The purpose of the paper is to show an integrated methodology based upon a survey campaign and natural observations both aimed to define Modal Share. The methodology has been applied to detect changes into the university community choices of transportation after the launch of a new sustainable mobility policy. 


\section{The integrated methodology for Modal Share definition}

The survey, named GOTOUNIBO, has been conducted online through the Qualtrics $\mathrm{xM}$ platform. The questionnaire is divided in 13 sections on habitual processes and attitudes on daily commuting home-university: prevalent mode of transportation, trip chain, parking habits and specific needs (i.e. Family related), satisfaction about the trip, institutional duty mobility, sharing mobility propensity, mobility apps usage (routing, gamification, info mobility features) and attitudes, ownership of personal transportation vehicles (mobility capital), opinions, perceived risk (road safety), more detailed questions about cars usage, socio-demography info.

The questionnaire has been addressed to the university community, staff, faculty, PhD students, lectures, administrative staff, students at any level. It's been submitted through several communication means. The survey lasted 20 days with a couple of reminders. GOTOUNIBO has been launched in 2017 and 2018.

These years has been very important for University of Bologna since they mark the passage from the mobility policy design to the implementation phase. The integrated model takes in account also observational evidence to reduce the conceptual survey's limitations and to verify the survey results. The observations have been focused on specific transportation means: car, bicycle, public transportation, train and walking. It has been established a quantitative check-list that enlist some items per categories.

The items for car are: number of car stationing in university parking lots and rotation rate. The rotation rate explains the daily number of cars parking on the same lot. The item for bicycles is the number of bikes present within university facilities and on public space at a radius of 350 meters (a distance that it's covered in less than 5-minutes walking, statistically used by public transportation as bus stop inter-distance).

The item for public transportation and train is the number of annual subsided tickets bought by students and staff. For bus only, it was also asked the number of drops-out of people near some relevant peripherical campuses. The question on walking habits is regarding the level of accessibility: it is defined as the number of architectural barriers present on walking paths from the bus stop. The observational evidence has been acquired through several visits on site by different teams of logistic experts, during the same period of the survey launch. Each team was composed by two specialists; to reduce the economic impact of the observations only campuses/schools more popular among students and staff were selected.

\section{Results and impact}

It has been obtained a wide difference between 2017 and 2018 samples consistency. In 2017, the complete responses were 4,432 versus 11,129 in 2018. The improvement was partly due by a more aggressive communication campaign in 2018. Secondarily, the new transportation policy adopted by the university has been more strongly perceived by users.

Focusing on bicycles, bus and car more easily subjected to variations, in 2017 the modal share detected for mono-modal trip has been (for all students and workers) $11 \%$ commuting home-University by bicycles, $7 \%$ by bus and $10 \%$ by car.

By the perspective of transport integration, it is relevant to underline that $48,9 \%$ of the sample is on mono-modal way, while $51,1 \%$ on multiple ways. It is also interesting to observe the articulation among species of trip chain (Figure 1 and 2). 


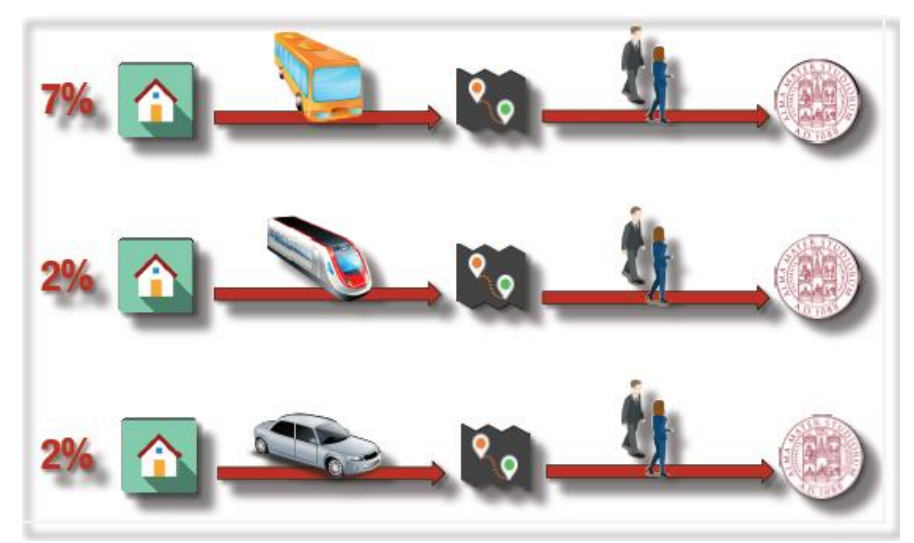

Figure 1 - Bi-modal trip chain modal share in 2017

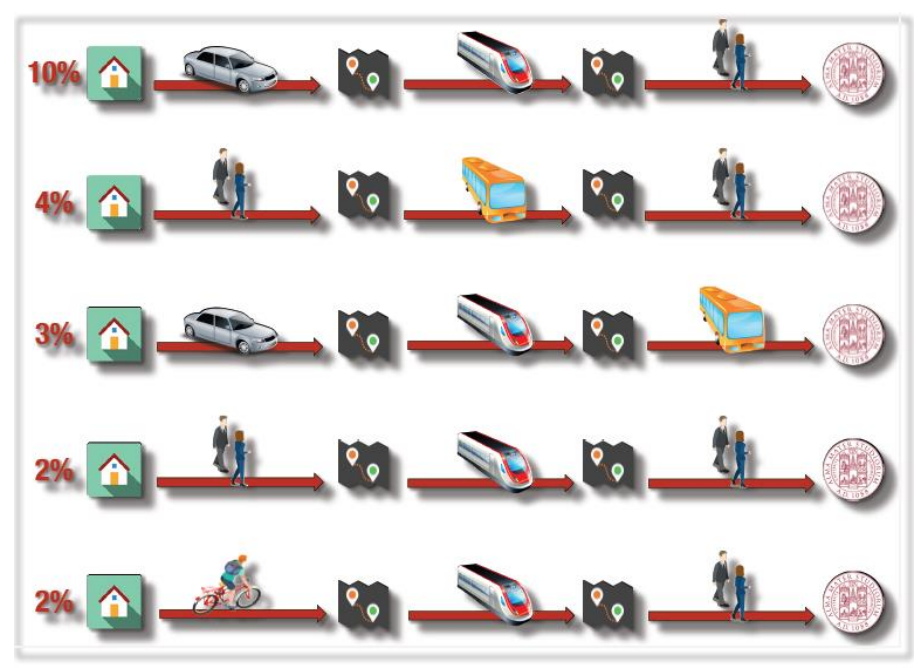

Figure 2 - Tri-modal trip chain modal share in 2017

In bi and tri-modal trip chain it is demonstrated that integration with bicycle and other transportation means was poor and needed improving measures. Most of these actions are relevant for Public Transportation only. Other improvements that the University of Bologna could put in place concerns the modal integration: the creation of point where it is possible to exchange mobility mode.

In general, the awareness of these modal shares had a direct impact on policy making, allowing the definition of a set of new sustainable measures aimed to act on bus and bike modal share:

1 Improving bicycles images as a real vehicle: the Almabike project. The project is based upon the design and marketing of 600 IOT connected bikes to be freely given to students in 2020. The 2017 project was promoted through a design contest for students followed by a co-working project between the winners and professional designers.

2 To increase the level of accessibility with 500 new parking slots for bikes in cities of Bologna and Cesena;

3 Endorsement of transport integration for students and staff: the university has signed an agreement with a private bike storage facility nearby the train station at a subsided price.

4 New public cycle-path design project, as an example of cooperation between the University of Bologna and the Municipality of Bologna to develop a connective bike lane from the very center to peripherical campuses in Bologna. 
Action taken for Bus support: The Freely moving project displays subsided annual ticket for urban buses. The investment of $724.000 €$ also permits to offer students two kinds of discount: $67 \%$ on the annual urban ticket subscription for 4.000 students (selected by a specific call) and another discount rate about $40 \%$ for all others students. The application of the measures registered in 2018 a new commuting home-University modal share: $24,0 \%$ by bicycles, $14,26 \%$ by bus and $10 \%$ by car. The trip chain basically remained the same.

It's interesting to underline the higher percentage of bike share, depending not only on the sensitive communication approach to Almabike project and the new bike parking facilities, but also on new cycle path. It should be noticed that women between 19 and 25year-old use bicycles less than man of the same age. $23 \%$ of our female students use bikes in comparison to $35 \%$ of males.

The survey also explains that female minor usage of bikes is related to safety issues (many new cycle paths present some unsafety crossroads or continuity lane interruption between different road sections) and trip comfort issues. The trend is confirmed in literature [17].

In parallel, the bus share confirms the increasing trend related to policy application.

The observational evidences in both 2017 and 2018 assessed the survey's data collection quality. The abovementioned modal shares were basically confirmed by the check-list analysis. The percentage subjected to real observation is about $40 \%$ of the university locations. Even considering the errors inherent to the location's variability, it possible to assume the correctness of the established modal share. In 2018, after the policy application, the number of annual subsided bus ticket raised to 8,000 , equal to the $10 \%$ of the university students. Adding the number of monthly tickets is easy to reach the estimated share of $14,2 \%$.

\section{Conclusions and recommendations}

The paper explores the transport related issue on modal share evaluation and its link with policy-making. Data was collected in 2017 and 2018, through a questionnaire submitted by the University of Bologna to its students and staff. The methodology has been integrated by observational evidence, obtained through the use of specialized teams with a check-list defined upon the most common detectable mobility variables. We have explored the effect of some university policy on transportation on modal share changing, and related issues on gender behavior.

Another important factor emerges for the above analysis.

Since February 2020, the Covid-19 spread-out has forced to change our personal habits; the post lock-down phase requires a new analysis on mobility and it is easy to deduce that some mandatory variables, such as social distancing demands a new sustainable mobility paradigm. In this uncertain scenario, it is more important to rely on affordable and consistent data collections criteria. The integrated methodology presented in this paper is a valid tool with no great economic efforts and a short development time. The integration between survey response and observational evidence obtained with an on-site check reduces common survey errors related to cognitive distortions and permits to evaluate the reliability of questionnaire responses.

In this post-Lockdown phase, the methodology could also help cities to balance between new transportation demand, adaptive mobility infrastructures and service offer. Further related research could be on the methodology for Decision Support System: by collecting data upon cyclists and bus user's through survey and observations, it could be 
possible to define a ranking of traditional routes transformable in emergency urban cycle paths during post-Lockdown phase, due to a less use of public transportation.

\section{References}

1. UN HABITAT State of the World's Cities 2010/2011 - Cities for All: Bridging the Urban Divide. EEA, 2013. Annual European Union Greenhouse Gas Inventory 1990-2011 and Inventory Report 2013.

2. Orimoloye I.R., et al.Implications of climate variability and change on urban and human health: A review Cities, 91 (2019), pp. 213-223

3. Lu L., Emerging study on the transmission of the Novel Coronavirus (COVID-19) from urban perspective: evidence from China CitiesVolume 103August 2020Article 102759

4. Mclean, H. Bulkeley, H.Crang, 2016. Negotiating the urban smart grid: socio-technical experimentation in the city of Austin. Urban studies, Volume 53, pp. 2346-3263.

5. Kern F., 2012. Using the multi-level perspective on social-technical transition to assess innovation policy. Technol. Forecast soc. Chabge, Volume 79, pp. 298-310.

6. Herold, D.M.; Lee, K.-H, 2017. Carbon management in the logistics and transportation sector: An overview and new research directions. Carbon Manag., Volume 3, pp. 1-19.

7. Urry J., 2004. The 'System' of Automobility. Theory Cult. Soc., Volume 21, pp. 25-39.

8. Greene David L., 2015. In: International Encyclopedia of the Social \& Behavioral Sciences (Second Edition).

9. Mohieldin M.,Vandycke N., 2017. Sustainable Mobility for the 21st Century, The World Bank. Available onlie at https://www.worldbank.org/en/news/feature/2017/07/10/sustainable-mobility-for-the21st-century), july.

10. Roggema R., Ermeed T.V., Dobbelsteen T., A.V.D, 2012. Incremental change, transition or transformation? Optimising change pathways for climate adaption in spatial planning. Sustain.For, pp. 2525-2549.

11. Canitez, F. 2019. Pathways to sustainable urban mobility in developing megacities: a social technical transition perspective, Technological Forecasting \& Social Change.

12. Banister, D. 2008. The sustainable mobility paradigm. Transp. Policy.

13. Klotildi S., 2014. Public Transport Integration: the Case Study of Thessaloniki, Greece. Mobil. TUM - Sustainable Mobility in Metropolitan Regions, May 19-20, 2014, Transport Research Procedia, Volume 4, pp. 535-552.

14. Gaskell G., Wright D., O'Murcheataigh, 1993. Reliability of survey. The Psychologist, Volume 6(11), pp. 500-503.

15. Schwartz, N. Hippler, H.G., Deutch, B., Strack, F., 1985. Response scale: effects of category range on reported behaviour and comparative judgments. Public Opinion Quarterly, pp. 49, 388-395.

16. Schwartz, N., Strack, F., Muller, G., Chassein, B., 1988. The range of response alterntives may determine the meaning of the question: further evidence on informative functions of response alternatives. Social Cognition, Volume 6, pp. 107-117.

17. Jan Garrard, Geoffrey Rose, Sing Kai Lo, 2008. Promoting transportation cycling for women: The role of bicycle infrastructure. Preventive Medicine, Volume 46(1), pp .55-9. 